\title{
CONNECTING ATOMISTIC AND EXPERIMENTAL ESTIMATES OF IDEAL STRENGTH
}

\author{
C. R. Krenn, ${ }^{1,3 *}$ D. Roundy, ${ }^{2,3}$ Marvin L. Cohen, ${ }^{2,3}$ D. C. Chrzan, ${ }^{1,3} \&$ J. W. Morris, Jr. ${ }^{1,3}$ \\ ${ }^{1}$ Department of Materials Science and Engineering, \\ University of California at Berkeley, Berkeley, California 94720, \\ ${ }^{2}$ Department of Physics, University of California at Berkeley, Berkeley, California 94720, \\ and \\ ${ }^{3}$ Materials Sciences Division, Lawrence Berkeley National Laboratory, \\ Berkeley, California 94720
}

\begin{abstract}
The ideal strength is the minimum stress required to plastically deform an infinite defectfree crystal and is an upper bound to the strength of a real crystal. Disturbingly, however, the best available experimental estimates of the ideal strengths of tungsten and molybdenum are $25-50 \%$ above the values predicted by recent ab initio density-functional calculations. This work resolves this discrepancy by extending the theoretical calculations to account for the triaxial state of stress seen in the nanoindentation experiments and by adjusting the experimental values to account for the crystallography of loading and the nonlinearity of the elastic response at large strains. Although an implicit assumption in many discussions of mechanical properties is that the ideal strength is not experimentally observable, as the true strength of most materials is limited by lattice defects, the close agreement between corrected experimental and theoretical estimates of ideal strength suggests that the ideal strength of some materials can be observed directly using nanoindentation.
\end{abstract}

PACS: 62.20.Dc, 62.20.Fe, 62.20.Qp. 


\section{Introduction}

If an infinite, defect-free solid is subjected to an increasing load, it deforms elastically until the stress exceeds the limit of elastic stability and irreversible deformation occurs. The elastic limit defines the "ideal strength" of the solid. Its value sets an upper bound on the mechanical strength the solid can have under the given load. ${ }^{1,2}$ Since the elastic limit is a property of the infinite, periodic lattice, it can be computed ab initio, with the consequence that the ideal strength is one of the few mechanical properties that are truly predictable.

The most immediately promising experimental approach to measuring the ideal strength is nanoindentation, in which an indenter with a tip radius of $50 \mathrm{~nm}$ to $1 \mu \mathrm{m}$ is pressed into the material surface. If the native defect density is low, the stressed volume beneath the small indenter may be defect-free. Moreover, since the shear component of the indentation stress reaches its maximum value some distance beneath the surface, deformation may initiate in the bulk. In this case, the local value of the stress required to initiate deformation is either the ideal shear strength or the stress required to nucleate dislocations homogeneously, a stress that should be very close to the ideal shear strength.

Encouragingly, very high values of the shear strength have been reported recently from nanoindentation studies of tungsten ${ }^{3,4}$ and molybdenum ${ }^{6}$. The peak shear stresses reported in $\mathrm{W}$ range from $25.7 \mathrm{GPa}^{3}$ with a $700 \mathrm{~nm}$ diamond tip to $28.6 \mathrm{GPa}^{4}$ with a 400 $\mathrm{nm}$ diamond tip. Peak stresses in Mo are reported to be $20 \mathrm{GPa}^{6}$ with a $50 \mathrm{~nm}$ diamond tip. These normalized strengths (16-18\% of the shear modulus) appear to be independent of the radius of the indenter. Surprisingly, however, the values reported are substantially above recent $a b$ initio calculations of the ideal shear strengths of these bcc metals. ${ }^{7,8}$ The work presented here resolves this discrepancy, and further establishes that under favor- 
able circumstances, one may observe experimental strengths approaching the ideal strength of a material.

The discrepancy is resolved by reanalyzing both theory and experiment. First, the theoretical solution must be modified to reproduce the geometry of the indentation load. The calculations reported in Refs. 7 and 8 assumed a fully relaxed shear load. However, the actual stress state at the point of maximum shear under the indenter is triaxial. This triaxial stress stabilizes the structure and raises the ideal strength in shear. Second, the experimental numbers must be corrected for the non-linearity in the stress-strain relation at finite strain, and also require a (smaller) correction to orient the shear onto the appropriate crystallographic plane. ${ }^{9}$ These corrections substantially lower the maximum shear stress that can be inferred from the experimental hardness data. The net effect is to remove the apparent discrepancy: to within the accuracy of our analysis, the measured shear strengths are either equal to or slightly below the computed ideal strengths, as they should be. Moreover, the difference between the measured shear strengths and the predictions of theory are now less than the uncertainties in the analysis $(<5 \%)$.

The next section of this paper presents the computational approach and results. This is followed by a discussion section and the conclusions.

\section{Computational Procedures and Results}

To quantify the effect of triaxial loading on the ideal strengths of W and Mo, we used the local density approximation (LDA) to density functional theory within an ultra-soft pseudopotential total-energy scheme $\mathrm{e}^{10,11}$ to calculate the stress-strain response for the active shear system in W and Mo $(<111>\{110\})$. (The calculations were done with the

VASP package. ${ }^{12-14}$ ) The stress states considered included relaxed simple shear (as in 
Ref. 7) and a triaxial stress determined numerically from the finite element modeling of nanoindentation described below. Using a plane-wave energy cut-off of 17 Ry with a Monkhorst-Pack $17 \times 17 \times 17 k$-point grid proved sufficient to achieve precision of better than $0.01 \mathrm{eV}$ in the calculated energies and better than $0.6 \mathrm{GPa}$ in peak stress. ${ }^{15}$ The triaxial stress increased the shear strength $\left(\tau_{\text {crit }}\right)$ of $\mathrm{W}$ from 20.0 to $22.7 \mathrm{GPa}$ and the strength of Mo from 17.1 to $18.2 \mathrm{GPa}$. (The unconstrained strength of $\mathrm{W}$ is higher than the value (18.2 GPa) calculated by Roundy et al., presumably because different pseudopotentials are used in the two calculations). Figure 1 shows the normalized stress-strain curves for $\mathrm{W}$ and Mo. They are similar with peak stresses near a shear strain of $17 \%$ as expected for the bcc structure. ${ }^{7}$

To correct the reported values of the experimental strengths we must examine how they are generated from the raw data. In Bahr et al.'s study of tungsten, ${ }^{4}$ a sharp diamond with a tip radius of approximately $400 \mathrm{~nm}$ is pressed into a single crystal with a polished surface. Yielding is marked by a sudden increase in the depth of penetration during loading. Prior to yielding, the load-displacement $(\mathrm{P}-\delta)$ response of the system is fit well by the Hertzian model of elastic contact: ${ }^{16}$

$$
P \propto \sqrt{R \delta^{3}}
$$

where $\mathrm{R}$ is the radius of the indenter tip. Since the stress is not measured directly, the Hertzian stress field is used to deduce the maximum shear stress underneath the indenter at the yield point. However, the Hertzian stress field assumes linear stress-strain behavior in both the indenter and the substrate. This assumption fails when the shear stress approaches the ideal strength (Fig. 1). 
To include non-linearity, a sinusoidal curve is fit to the $a b$ initio calculated stress-strain relation in Fig. 1. The sinusoid is then used as the elastic-plastic constitutive relation in a finite-element (FEM) model of nanoindentation. The 2D axisymmetric FEM model resembles an earlier model by Tang and Arnell ${ }^{17}$ and uses the ANSYS code. A frictionless sphere with a radius of 100 units is pressed into a $1600 \times 1600$ unit substrate. The sphere is meshed with elements approximately 2.75 units square, and the mesh on the substrate is refined from 100 unit square elements at the opposing boundaries to 1.25 unit square elements directly beneath the indenter. In the model, a stiff indenter is pressed into both a linear-elastic substrate and into a substrate with the stress-strain response given in Fig. 1. The shear modulus of the indenter is taken to be 1000 times that of the substrate, and both indenter and substrate are assumed elastically isotropic with Poisson's ratios of 0.3 .

We specifically considered indentation normal to a $\langle 100\rangle$ surface, which is the strongest configuration, and calculated the resolved shear stress on the most favorably oriented member of the $<111>\{110\}$ slip system. (This plane should be the first to shear.) Figure 2 shows the calculated stress contours for a linear-elastic solid (2a) and for a solid with the non-linear constitutive relation given in Fig. 1 (2b). As expected, ${ }^{18}$ in both cases the maximum value of the resolved shear stress is located beneath the surface slightly off the loading axis. Fig. 3 presents plots of the applied stress (3a) and the maximum shear stress (3b) as functions of the indentation depth. The classic Hertzian model reproduces the overall load-displacement curve of the nonlinear substrate very well (3a), but significantly overestimates the maximum shear stress in the non-linear solid at the experimental yield point (3b). (The Hertzian model also slightly misestimates the stress in the linearelastic solid, apparently because the assumption that the contact area is small compared to the size of the elastic bodies in contact $^{16}$ fails for loads near the yield point.) The Hertzian value for the critical resolved shear stress on the $\langle 111\rangle\{110\}$ slip system at the 
experimentally observed failure depth (the indentation depth $\delta$ normalized by the indentor radius $R=0.16$ ) overestimates the peak stress in the non-linear substrate by a factor of 1.08. The Hertzian value for the maximum shear stress at failure (which is the value reported in Refs. ${ }^{4}$ and ${ }^{6}$ ) is above the true non-linear peak stress by a factor of 1.21 . This means that the maximum shear stresses cited in past analyses are approximately $20 \%$ higher than the actual stresses seen on the weakest slip systems, where failure is likely to initiate.

Additional FEM modeling shows that the correction factors are not particularly sensitive to the conditions of the model. Using a Poisson's ratio of 0.2 for the indenter (the experimental value of diamond) and Poisson's ratios of 0.25 and 0.35 (typical of most metals) gave correction values of 1.20 and 1.23. If we account for the compliance of the diamond indenter on $\mathrm{W}$ the total error introduced by using the Hertzian stress and ignoring crystallography decreases to a factor of 1.17. Raising the coefficient of friction between the indenter and the substrate to 0.4 reduces the factor to 1.20 .

Figure 4 summarizes the effects of the triaxiality of loading and of the non-linear elastic correction. The error bar on the corrected experimental numbers includes the possible effects of friction, of varying the ratio of the substrate and indenter's stiffnesses, and of varying the Poisson's ratios of the substrate and indenter. When all corrections are properly made, the maximum shear strengths that can be inferred from nanoindentation experiments on $\mathrm{W}^{4}(22.8-24.0 \mathrm{GPa})$ and $\mathrm{Mo}^{6}(16.0-16.8 \mathrm{GPa})$ are, to within the accuracy of our analysis, either equal to or below the theoretical values of the ideal strength ( $\mathrm{W}=$ 22.1-23.3 GPa; $\mathrm{Mo}=17.6-18.8 \mathrm{GPa}$ ), as they should be. At the same time, the theoretical and experimental values are reasonably close, which suggests that nanoindentation may provide a viable means for measuring ideal strength. 


\section{Discussion}

\section{Other sources of error}

We note that the peak shear stresses inferred from the nano-indentation experiments on W and Mo are only $96 \%$ of the absolute peak stresses predicted by FEM modeling. Failure in the FEM model occurs when the slope of the peak stress-strain curve (Fig. 3b) drops to 0 , which occurs at a relative indentation depth $(\delta / R)$ of $\sim 0.21$. However, failure for both $\mathrm{W}$ and Mo occur for relative indentation depths of 0.15-0.17. If indentation continued to a relative depth of 0.21 , the peak stress would be a factor of 1.04 $(=1 / \operatorname{Sin}(\pi \times 0.16 / 0.21 / 2)$ higher.

In the calculations presented here, we assumed elastic isotropy because that made the problem computationally tractable using the resources that we had available. In fact, the shear response of a real bcc material is stiffer than the sinusoidal form we have assumed when shear is applied in directions other than 111. The maximum effect that this could have on our analysis can be estimated by comparing the peak strains at the indentation failure depth for elastic and plastic cases. The peak shear strain for the linear-elastic case $(6.2 \%)$ is $10 \%$ lower than the non-linear elastic case $(6.9 \%)$. Given that the anisotropic solution will lie between the linear and non-linear cases and given that the weakest slip system still has a sinusoidal stress-strain response, a gross lower bound on the anisotropic peak stress solution would be $5 \%$ lower than the isotropic case. However, since the stressstrain behavior on the weak slip system will be identical for the isotropic and anisotropic solutions, the true correction is likely to be much less than $5 \%$.

\section{Other models of the limits of indentation strength}

A recent paper by Kramer et $a l^{3}$ uses atomic force microscopy to demonstrate that limited (and, on some occasions, reversible) plastic deformation can occur before bulk 
yielding is detected in a load-displacement curve. This implies that a small number of dislocations can either move or nucleate before bulk plasticity is observed. Kramer et al. further argue that the beginning of large-scale plasticity is limited by the fracture strength of a surface oxide layer. This may be a reasonable explanation for the observed increase in the yielding load of $\mathrm{Fe}-3 \% \mathrm{Si}$ single crystals with increasing oxide thickness, ${ }^{4}$ but it cannot convincingly explain the apparent insensitivity of yield load to oxide thickness in a series of experiments on tungsten single crystals performed by the same authors. ${ }^{4}$

However, the observations of Kramer et al. ${ }^{3}$ may also imply that the onset of macroscopic plasticity is limited by the activation of a dislocation multiplication mechanism. In the absence of such a mechanism, a sharp diamond tip loading a flaw-free region of a metallic surface could nucleate a small number of dislocation loops. Nucleation could occur either at the surface or at an interface between a surface oxide and the bulk metal. These loops will be pinned at the surface but can grow under increasing applied load. If the growing loops did not encounter obstacles during their growth, this plastic deformation would be reversible. If the collapsing loops are pinned by obstacles, there will be a residual deformation on the surface, as Kramer et al. have observed. Time dependent recovery is possible if thermal activation and the line tension of the loops is sufficient to overcome the obstacles.

A macroscopic yield point requires either a renewable dislocation source beneath the surface (see Ref. 5, Chapter 20, for examples) or the homogeneous nucleation of a shower of dislocations in the bulk. We can estimate the dislocation density that can be released by an elastic instability by equating the elastic strain energy per unit volume $\left(=(1 / 2) G \gamma^{2}\right)$ with the line energy of a given line density of dislocations $\left(\sim(1 / 2) G b^{2} \rho\right)$. For any shear modulus $G$, a strain $\gamma$ of only 0.01 , and a burger's vector $b$ of $3 \AA$, we get a dislocation 
density $\rho$ of about $10^{15} / \mathrm{m}^{2}$. Furthermore, the energy released in the instability is more than sufficient to fracture a thin surface oxide film.

\section{Conclusions}

After incorporating the triaxial state of stress seen in nanoindentation experiments into $a b$ initio calculations of ideal strength and after properly accounting for the crystallography of loading and the nonlinearity of the elastic response at large strains in the analysis of experimental nanoindentation experiments, we find close agreement between corrected theoretical and experimental estimates of the ideal strengths of W and Mo. This suggests that the ideal strength of some materials can be observed directly using nanoindentation.

\section{Acknowledgements}

The authors acknowledge insightful conversations with W.D. Nix. The ab initio calculations were performed using the total-energy and molecular-dynamics program VASP (Vienna ab initio simulation program) and ultra-soft pseudopotentials developed at the Institut für Theoretische Physik of the Technische Universität Wien. This work was supported by the Director, Office of Energy Research, Office of Basic Energy Sciences, Materials Sciences Division of the U.S. Department of Energy.

\section{References}

* Current address: Lawrence Livermore National Laboratory, University of California, Livermore, California 94550

Electronic address: entremment.gov

1 A. Kelly and N. H. Macmillan, Strong Solids (Clarendon Press, Oxford, 1986). 
J. W. Morris, Jr., C. R. Krenn, D. Roundy, and M. L. Cohen, in Phase Transformations and Evolution in Materials, edited by P. E. A. Turchi and A. Gonis (The Minerals, Metals and Materials Society (TMS), Warrendale, PA, 2000), p. 187.

D. E. Kramer, K. B. Yoder, and W. W. Gerberich, Phil. Mag. A 81, 2033 (2001).

D. F. Bahr, D. E. Kramer, and W. W. Gerberich, Acta Mater. 46, 3605 (1998).

J. P. Hirth and J. Lothe, Theory of Dislocations (Wiley, New York, 1982).

W. D. Nix, private communication, (Stanford University, 2001).

D. Roundy, C. R. Krenn, M. L. Cohen, and J. W. Morris, Jr., Phil. Mag. A 81, 1725 (2001).

D. Roundy and M. L. Cohen, 2001).

The crystallographic correction was included in a more recent presentation by Nix et al.: W. D. Nix, R. Saha, E. T. Lilleodden, D. Barbero, B. M. Clemens, paper presented at the Fall Materials Research Society Meeting, Boston, MA, 30, November 2000.

M. L. Cohen, Phys. Scr. T1, 5 (1982).

J. Ihm, A. Zunger, and M. L. Cohen, J. Phys. C 12, 4409 (1979).

G. Kresse and J. Hafner, Phys. Rev. B 49, 14251 (1994).

G. Kresse and J. Furthmüller, Comput. Mater. Sci. 6, 15 (1996).

G. Kresse and J. Furthmüller, Phys. Rev. B 54, 11169 (1996).

The computed bulk modulus (331 GPa) and lattice constant (3.12 A) agree very well with recent full potential calculations that used the local density approximation (335 GPa and 3.13 A): S. Kurth, J. P. Perdew, and P. Blaha, Int. J. Quantum Chem. 75, 889 (1999).

K. L. Johnson, Contact Mechanics (Cambridge University Press, Cambridge, 1985).

K. C. Tang and R. D. Arnell, Thin Solid Films 355-356, 263 (1999). 
J. A. Zimmerman, P. A. Klein, and S. M. Foiles, in Fundamentals of Nanoindentation and Nanotribology II, edited by S.P. Baker, R.F. Cook, S.G. Corcoran and N. R. Moody (Materials Research Society, Warrendale, PA, 2001). 


\section{Figure Legends}

Figure 1 Ab initio stress-strain response for $\langle 111>\{110\}$ slip in tungsten and molybdenum. Data is shown for both simple shear and shear along a path that includes the triaxial stress states seen during indentation. The solid line is the sinusoidal constitutive relation used in our FEM modeling.

Figure 2 Contour plots of the maximum $<111>\{110\}$ shear stresses beneath a rigid spherical indenter near the failure loads. (a) pictures a material with linear-elastic behavior, and (b) a material with the non-linear behavior shown in Fig. 1. The stress is normalized to the peak stress in (b) (=.089 of the shear modulus, $G)$.

Figure 3 Macro- and micro-scopic response of the linear and non-linear FEM models and the analytic Hertzian solution. (a) Normalized applied stress as a function of indentation distance. The normalized stress is $P /\left(G \pi R^{2}\right)$, where $P$ is the applied load, $R$ is the radius of the indenter, and $G$ is the shear modulus of the indenter. (b) Maximum shear stress and maximum shear stress resolved on $\langle 111>\{110\}$ as a function of indentation depth. The $\times$ and its error bar marks the upper range of indentation depths at which experimental failure occurred in W and Mo.

Figure 4 Comparison between theoretical and experimental estimates of ideal strength. Experimental estimates of ideal strength for both tungsten ${ }^{4}$ and molybdenum ${ }^{6}$ based on a Hertzian contact model exceed $a b$ initio theoretical calculations under loading conditions of simple shear. However, ab initio calculations that include the effects of multiaxial constraint during indentation slightly exceed experimental estimates which incorporate both the crystallography of slip and the non-linear elastic response expected near the limit of elastic stability. 


\section{Figures}

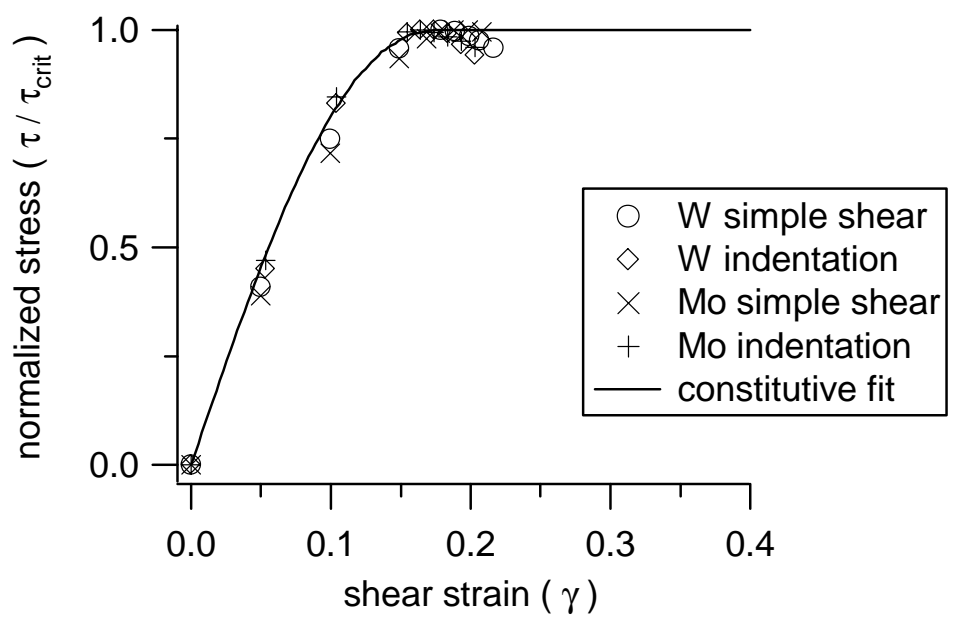

Figure 1 Ab initio stress-strain response for $<111>\{110\}$ slip in tungsten and molybdenum. Data is shown for both simple shear and shear along a path that includes the triaxial stress states seen during indentation. The solid line is the sinusoidal constitutive relation used in our FEM modeling. 

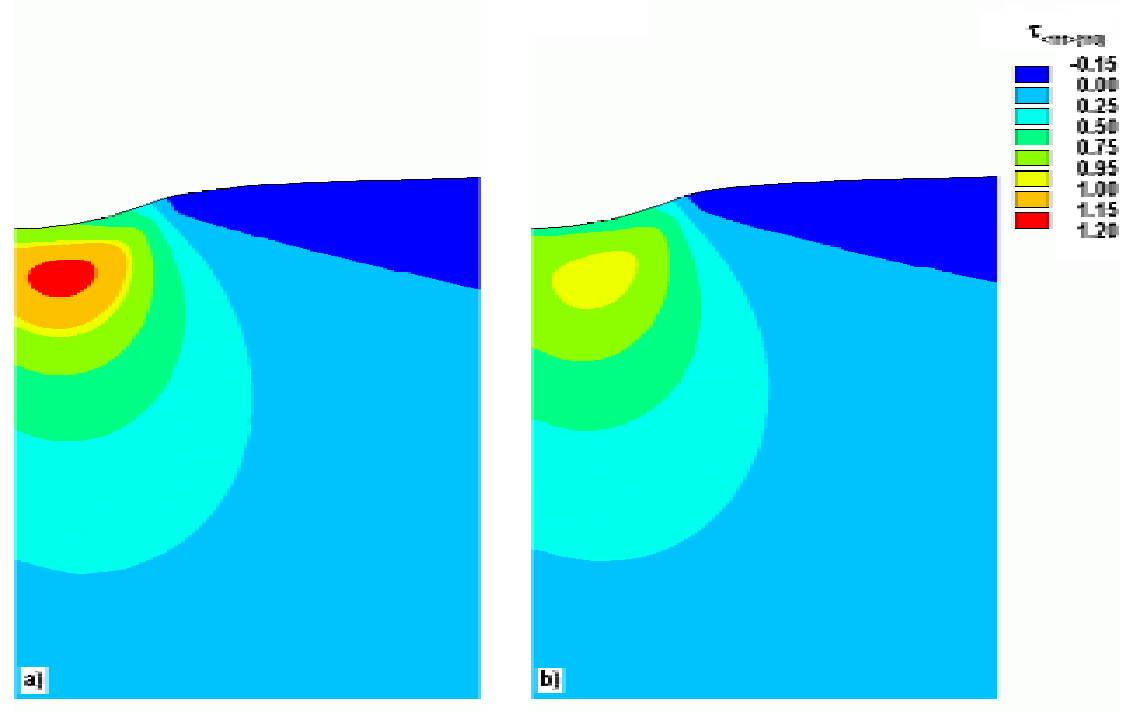

Figure 2 Contour plots of the maximum $<111>\{110\}$ shear stresses beneath a rigid spherical indenter near the failure loads. (a) pictures a material with linear-elastic behavior, and (b) a material with the non-linear behavior shown in Fig. 1. The stress is normalized to the peak stress in (b) (=.089 of the shear modulus, $G)$. 

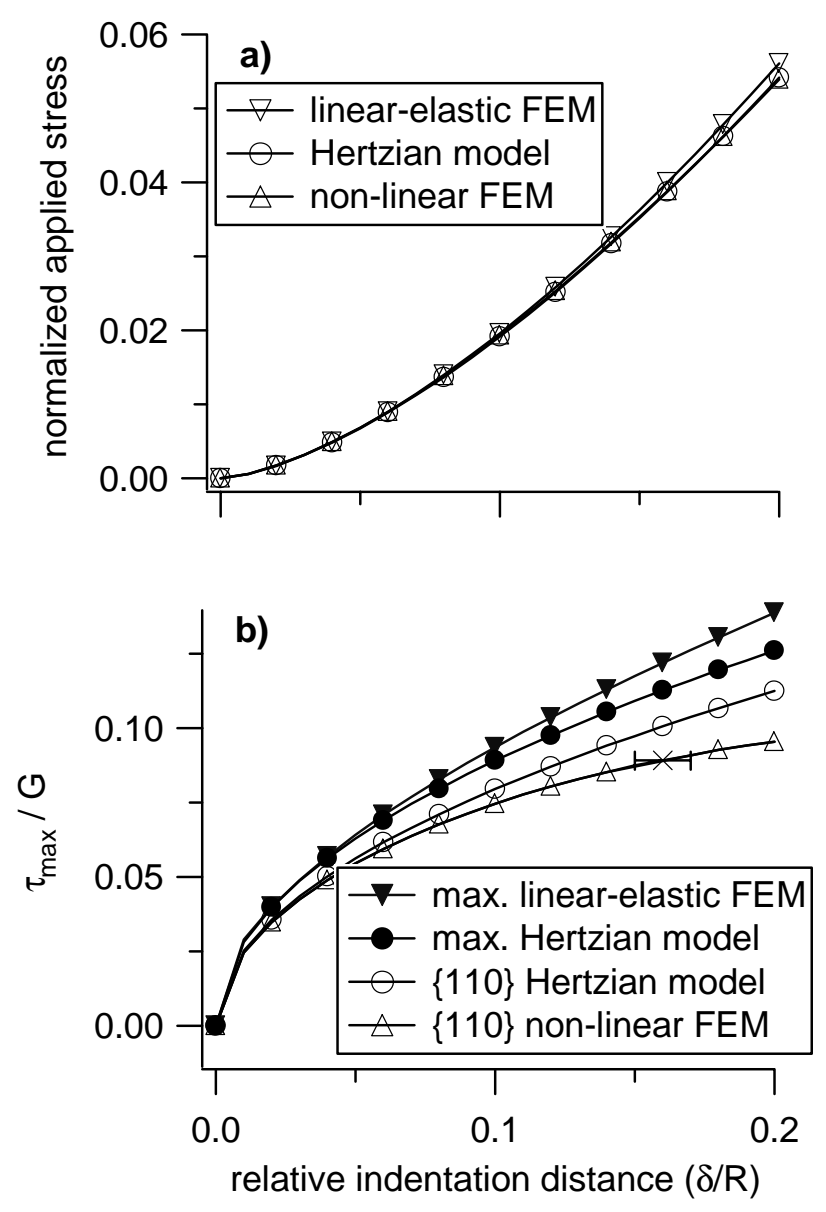

Figure 3 Macro- and micro-scopic response of the linear and non-linear FEM models and the analytic Hertzian solution. (a) Normalized applied stress as a function of indentation distance. The normalized stress is $P /\left(G \pi R^{2}\right)$, where $P$ is the applied load, $R$ is the radius of the indenter, and $G$ is the shear modulus of the indenter. (b) Maximum shear stress and maximum shear stress resolved on $<111>\{110\}$ as a function of indentation depth. The $\times$ and its error bar marks the upper range of indentation depths at which experimental failure occurred in W and Mo. 


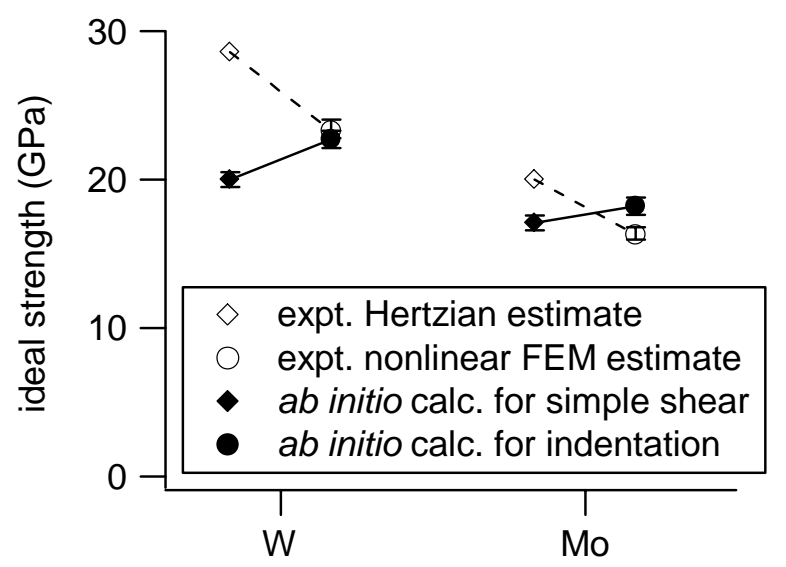

Figure 4 Comparison between theoretical and experimental estimates of ideal strength. Experimental estimates of ideal strength for both tungsten ${ }^{4}$ and molybdenum ${ }^{6}$ based on a Hertzian contact model exceed ab initio theoretical calculations under loading conditions of simple shear. However, ab initio calculations that include the effects of multiaxial constraint during indentation slightly exceed experimental estimates that incorporate both the crystallography of slip and the non-linear elastic response expected near the limit of elastic stability. 